\title{
Make or break - Automotive business models at crossroads
}

Ralf Kalmbach, Bain \& Company Germany, Inc.

This manuscript is not available according to publishing restriction.

Thank you for your understanding. 